\title{
Estrategias docentes digitales: una aproximación desde la Didáctica
}

Diana Marín Suelves. Universitat de València

Mercedes Romero Rodrigo. Universidad Antonio de Nebrija

José Peirats Chacón. Universitat de València
(iD) $0000-0002-5346-8665$

(iD) $0000-0002-9103-5668$

(D) $0000-0002-6580-2712$

Recepción: 09/01/2019 | Aceptado: 16/01/2019

Correspondencia a través de ORCID: Diana Marín

iD $0000-0002-5346-8665$

Citar: Marín, D, Romero, MM y Peirats, J (2019). Estrategias docentes digitales: una aproximación desde la Didáctica. REIDOCREA, 8(2), 1-5.

\begin{abstract}
Resumen: La sociedad actual, caracterizada por el impacto de la tecnología en los diferentes ámbitos de la vida cotidiana, construye nuevas formas de hacer, de vivir, de ser, de interactuar, de conocer y de aprender. En este contexto, la escuela no queda al margen de este proceso de cambio e innovación y se requiere de la puesta en práctica de estrategias didácticas digitales para atender a las características y demandas del alumnado que escolariza. Sobre ello y, a partir de los resúmenes de los artículos que componen el monográfico, se realiza un análisis de contenido que ha permitido identificar las palabras de uso frecuente en el ámbito analizado, empleando la herramienta Atlas.ti.8 para la representación de los resultados.
\end{abstract}

Palabras clave: Estrategias educativas | Digitalización

Digital teaching strategies: an approach from the Didactic

\begin{abstract}
The society that we are currently living in can be understood on the basis of the technology that impacts in our daily life, which builds new ways of doing, of living, of being, of interacting, of knowing and learning. This context of change and innovation influences both primary and secondary education, and different digital teaching strategies have to be applied to fulfill the needs and characteristics of the pupils. This paper analyzes the content of the abstracts of the papers that make up the monograph. It identifies the words that are frequently used in the referred field, by using the Atlas.ti. 8 software for the representation of the results.
\end{abstract}

Keywords: Educational strategies | Digitalization

Una de las características inherentes a nuestro tiempo y que, con bastante probabilidad lo definen a la perfección, tiene que ver con el hecho de vivir en continuo cambio. Nada parece servir para perdurar, y la necesidad de innovar se incorpora, con mayor o menor acierto, en nuestro quehacer y, atendiendo a lo que nos ocupa, en nuestras prácticas educativas. Consecuentemente, no será casual que se nos apremie a preparar al alumnado para vivir en un contexto de constante cambio, donde la capacidad de adaptación y evolución son competencias irrecusables en la formación de la ciudadanía del mañana.

Inmersos en este bucle constante, el papel de la tecnología en la progresiva o, mejor dicho, exponencial transformación digital de nuestra sociedad, como si de un portaestandarte se tratase, marca el paso en multitud de ámbitos y entre ellos el de los centros docentes. Actualmente, según la Subdirección General de Estadística y Estudios del Ministerio de Educación y Formación Profesional (2018) el 86.5\% de los centros educativos cuenta con página web publicada en internet, el $40.1 \%$ ofrecen servicios a través de un Entorno Virtual de Aprendizaje (EVA) y la mitad (50.9\%) cuenta con servicios en la nube.

Asumidos estos datos por institucionales, coincidimos con Area (2017) en afirmar que la mediación tecnológica y la transformación organizacional trasgrede los marcos tradicionales, mutando sus fronteras hacia la liquidez y ubicuidad, flexibilizando y diversificando tanto los espacios como los tiempos y sus dinámicas. Lo que proporciona 
a la comunidad educativa, pero en especial al profesorado, la oportunidad de acercarse a otros profesionales con inquietudes e intereses comunes más allá de su entorno cercano, pero también al alumnado y a sus familias gracias en gran parte, a la integración tecnológica en sus prácticas educativas cotidianas (Gabarda, Alonso y Boronat, 2017; Moreno, Pardo y Marín, 2018). De hecho, en esta línea, convenimos en señalar como el propio concepto de 'TIC' ha quedado superado por las 'TAC' (Tecnologías del Aprendizaje y del Conocimiento) o 'TEP' (Tecnologías para el Empoderamiento y la Participación), poniendo el foco precisamente en el papel activo de la comunidad, más allá del acceso y consumo de información (Granados, López, Avello, Luna, Luna y Luna, 2014).

Volviendo la vista atrás, no hace tanto tiempo que los esfuerzos de los gobiernos se centraban en la dotación de equipamiento informático en los centros, así como en la conectividad de los mismos lo que supuso, además, la creación de plataformas institucionales (Santana, Eirín y Marín, 2017). Esta etapa caracterizada por las mejoras en el acceso y uso de la informática le siguió, ya en la década del 2000 , un periodo donde el foco de atención acabó situándose en los esfuerzos por lograr la integración digital pedagógica (Romero, Peirats, San Martín y Gallardo, 2014). Si nos fijamos en la normativa, a pesar de ser la LOGSE (1990) la primera ley con alusiones directas a las TIC, no fue hasta la LOE (2009) donde se acometió un mayor desarrollo. Avance que se mantuvo con la ley educativa vigente, la LOMCE (2013), respetando de forma general los planteamientos de su antecesora en sus artículos 111, 112 y 157 e introduciendo en su preámbulo, como novedoso, la consideración de las TIC como una herramienta clave en la formación del profesorado.

Hoy por hoy, en plena era de aprendizaje social mediado por las TIC, opciones metodológicas como la gamificación, flipped classroom o herramientas como la realidad virtual, los videoblogs o la realidad aumentada, la programación o la robótica educativa cada vez son más frecuentes en nuestras aulas. Señalamos, por tanto, que la competencia digital de los docentes ante estas innovaciones debe ser el primer paso sine qua non para poder acometer la evolución digital de nuestras escuelas con garantías de éxito. Sobre esto, la Red Universitaria de Tecnología Educativa (RUTE) publicó hace una década una declaración sobre formación y desarrollo de las competencias de los futuros profesores para el uso pedagógico de las TIC, situando el foco de interés en la formación inicial, sin que ésta se centrase exclusivamente en el manejo de los diferentes desarrollos y que propiciase el conocimiento necesario para ser capaces de integrarlas en la práctica escolar. Lo que se ha traducido en estudios recientes centrados en analizar el desarrollo de la competencia digital docente en los diferentes planes de estudio (Peirats, Marín, Granados y Morote, 2018) de los Grados de Maestro (Martín y Cepeda, 2017) o postgrados como el Máster de Profesor de Secundaria, y en un aumento de la preocupación del papel de la didáctica en relación a la utilización de los artefactos digitales en las aulas, lo que nos ha llevado a la iniciativa de acometer el proyecto que presentamos en esta revista.

Tras estas breves líneas introductorias sobre la formación y el uso de las tecnologías en las prácticas educativas del profesorado en los centros describimos, a continuación, el conjunto de trabajos que presentamos a su consideración, esperando que su lectura les depare el mismo interés y satisfacción que a nosotros nos han producido. Por tanto, el presente monográfico está compuesto por diferentes artículos basados en investigaciones, reflexiones, revisiones de la literatura, experiencias y buenas prácticas docentes relacionadas con el proceso de digitalización educativa. Y que son resultado, muchas de ellas, de proyectos de investigación financiados por entidades públicas autonómicas y nacionales. En relación a su autoría, la mayoría son aportaciones de investigadores de diferentes universidades, miembros de reconocidos grupos de 
investigación como son CRIE de la Universitat de València, EDULAB de la Universidad de La Laguna o STELLAE de la Universidad de Santiago de Compostela.

A partir de los títulos, palabras clave y resúmenes de los artículos que componen el monográfico hemos realizado una nube de palabras (Figura 1) con el software específico para análisis cualitativos de datos Atlas.ti 8 , lo que nos ha permitido identificar los grandes temas que se van a tratar en este número, tras eliminar palabras de las categorías de adverbios, nexos, artículos y monosílabos, en una nube resultante con un umbral de 4 en frecuencia y un máximo de 24 veces. Las palabras más frecuentes son: estrategias (24), digitales (21), profesorado (18), recursos (16), aprendizaje (15), docentes (14), primaria (13) o educación (12).

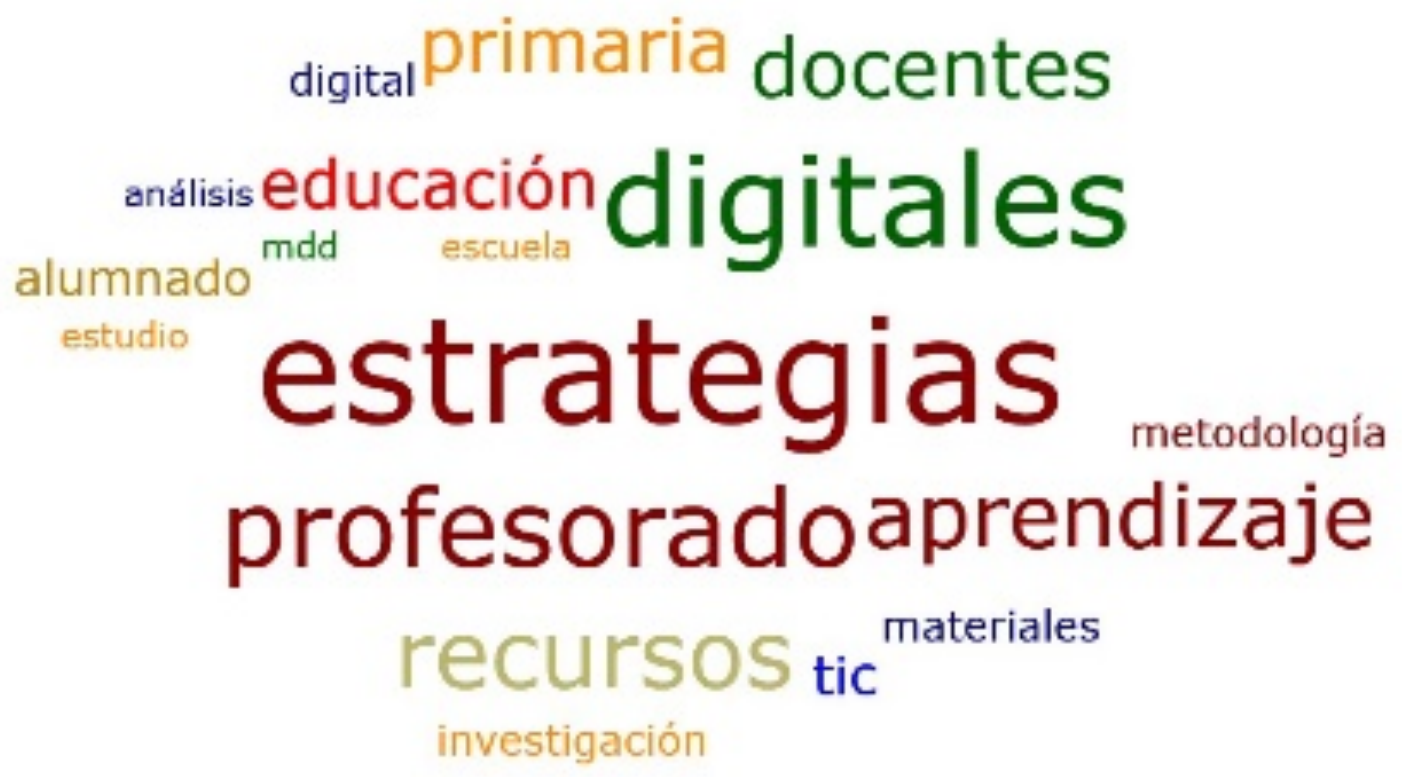

Figura 1. Nube de palabras frecuentes en los resúmenes del monográfico.

Como se puede observar, emergen como principales temas el de las estrategias y el de lo digital, que sumados sus valores al de los docentes ocupan más de la mitad del total, lo que corresponde con el objeto de nuestras pretensiones en este monográfico, compuesto con una serie de textos que dan respuesta a cuestiones de gran calado en la intersección de la didáctica con la tecnología, tales como son: la tendencia en la investigación sobre TAC en general y sobre las estrategias docentes digitales en particular, el uso de las tecnologías en la atención a la diversidad, la aplicación de estrategias docentes mediadas por tecnología, el empleo de materiales didácticos digitales, el papel de la tecnología en el desarrollo de las competencias clave, los recursos ludificados o el enfoque adoptado en la formación del profesorado.

En cuanto a la organización de este número, en el primer artículo Isabel Pardo y Ángel San Martín, de la Universitat de València y Edisson Cuervo de la Universidad de Antioquia (Colombia) reflexionan sobre la lógica que subyace al uso de estrategias docentes/discentes en la escuela y sobre la redefinición del trabajo pedagógico, desde la aceptación de la hibridación entre lo virtual y lo experimentado.

Si nos situamos en las estrategias didácticas del profesorado en formación, Vicente Gabarda, Ernesto Colomo y Mercedes Romero, de la Universidad Internacional de Valencia, disertan acerca de las potencialidades del modelo de educación online como facilitador del aprendizaje del futuro maestro, analizando algunas propuestas 
metodológicas para llevar a cabo procesos de enseñanza y aprendizaje que fomenten el aprendizaje colaborativo y la construcción crítica del conocimiento.

Seguidamente Diana Marín e Isabel Vidal (Universitat de València) realizan un análisis bibliométrico de la producción científica presente en la base de datos Scopus para conocer las tendencias de la investigación en el campo de las Tecnologías del Aprendizaje y el Conocimiento (TAC).

Por otra parte, Isabel María Gallardo y Héctor Saiz (Universitat de València) describen, a continuación, las estrategias y métodos que las tecnologías de la información y la comunicación posibilitan en el proceso de transición entre etapas educativas en relación a la formación del profesorado.

Y en el siguiente artículo, Ana de Castro y Abraham Cerveró (Florida Universitaria) estudian la percepción de la incidencia de la plataforma Open Moodle, los MOOC y los intercambios virtuales Erasmus en el desarrollo de sus competencias didácticas desde la perspectiva del alumnado del Grado de Educación Primaria.

Trasladándonos a otros contextos autonómicos, Carlos González y Sebastián Martín (Universidad de La Laguna) describen las estrategias metodológicas llevadas a cabo por los docentes tras la implantación del programa Escuela 2.0 en cuatro centros educativos de Primaria situados en Canarias.

A continuación, Montse Castro y Oscar Mallón (Universidade da Coruña) analizan la implementación del aula invertida para el desarrollo de la empatía del alumnado de los últimos cursos de Primaria como medida de atención a la diversidad.

Y, por su parte, María Belén San Nicolás y Anabel Bethencourt (Universidad de La Laguna) presentan un estudio cualitativo centrado en el análisis de la visión del profesorado sobre los materiales didácticos digitales, identificando tanto las potencialidades como las dificultades para su puesta en práctica.

Cerrando este monográfico Irene Moya-Mata y José Peirats Chacón, de la Universitat de València presentan los resultados de un estudio de revisión bibliográfica en el que analiza la literatura científica sobre el aprendizaje basado en proyectos en el área de Educación Física.

\section{Referencias}

Area, M (2017). La metamorfosis digital del material didáctico tras el paréntesis Gutenberg/The digital metamorphosis of didactic material after the parenthesis Gutenberg. Revista Latinoamericana de Tecnología Educativa-RELATEC, 16(2), 13-28.

Gabarda, V, Alonso, I y Boronat, J (2017). ClassDojo: una experiencia de aula. Comunicación y pedagogía: Nuevas tecnologías y recursos didácticos, 299, 64-70.

Granados, J, López, R, Avello, R, Luna, D, Luna, E y Luna, W (2014). Las tecnologías de la información y las comunicaciones, las del aprendizaje y del conocimiento y las tecnologías para el empoderamiento y la participación como instrumentos de apoyo al docente de la universidad del siglo XXI. Medisur [revista en Internet]. 12(1), [aprox. 5 p.]. Disponible en: http://ir.uv.es/eBgO4uH
Martín, S y Cepeda, O (2017). Las TIC en el grado de maestro/a de Primaria. Comunicación y pedagogía: Nuevas tecnologías y recursos didácticos, 299, 51-58.

Moreno, E, Pardo, Ml y Marín, D (2018). Tecnologies a l'escola per millorar la comunicació. Guix: Elements d'acció educativa, 442, 27-31.

Peirats, J, Marín, D, Granados, J y Morote, D (2018) Competencia digital en los planes de estudios de universidades públicas españolas. REDU: Revista de Docencia Universitaria, 16(1), 4.

Romero, MM, Peirats, J, San Martín, A y Gallardo, IM (2014). Percepciones en torno al coordinador TIC en los centros Educativos Inteligentes. Un estudio de caso. Educar, 50(1), 167-184. 
Santana, P, Eirín, R y Marín, D (2017). Análisis y evaluación de portales institucionales en España. Los casos de Canarias, Galicia y Valencia. RELATEC: Revista Latinoamericana de Tecnología Educativa, 16(2), 29-48.
Subdirección General de Estadística y Estudios del Ministerio de Educación y Formación Profesional (2018). Enseñanzas no universitarias. Sociedad de la información y la comunicación en los centros educativos 2016/2017. Recuperado de http://ir.uv.es/w30IORS 